\title{
Plataforma computacional para la generación de actividades educativas a través de la herramienta Mimio Teach
}

\section{Computational platform for the generation of educational activities through the Mimio Teach tool}

\section{Diana Lancheros Cuesta ${ }^{1}$, Mayra Daniela Vargas ${ }^{2}$}

${ }^{1}$ Docente Investigador, Grupo de Investigación AVARC, Universidad de La Salle, Carrera 2-Calle 10, Bogotá, Colombia dilancheros@unisalle.edu.co

2 Ingeniera en Automatización, Grupo de Investigación AVARC, Universidad de La Salle, Carrera 2-Calle 10, Bogotá, Colombia, vmayra14@unisalle.edu.co

Recibido: 15/ago/2019 - Revisado: 30/sep/2019

Aceptado: 30/oct/2019 - Publicado: 30/jun/2019

Resumen Los sistemas de recomendaciones son modelos computacionales que le permiten al usuario escoger las mejores opciones a nivel de contenidos y preferencias. Teniendo en cuenta lo anterior se desarrolló una aplicación para niños de 6 a 8 años, que permite generar recomendaciones de actividades didácticas, de acuerdo a la destreza psicomotriz del estudiante al interactuar con la herramienta tecnológica Mimio Teach. Las validaciones se llevaron a cabo teniendo en cuenta una asesoria psicológica para ser aplicado en niños con problemas de dyslexia.

Palabras claves sistemas de recomendación; adaptación; habilidades psicomotoras;

Abstract The recommendation systems are computer models that allow the user to select the best options at the level of content and preferences. Taking into account the above has become an application for children from 6 to 8 years, which allows us to generate recommendations for teaching activities, taking into account a student profile that psychomotor skill, interacting with the technological tool Mimio Teach.

Keywords recommendation system; adaptation; psychomotor skills; educational software. 


\section{Introducción}

Las dificultades en el aprendizaje son definidas como "un trastorno en uno o más procesos psicológicos involucrados en la comprensión o uso del lenguaje hablado o escrito, que puede manifestarse en una imperfecta capacidad de escuchar, pensar, hablar, leer, escribir, deletrear o hacer cálculos matemáticos, incluyendo condiciones tales como discapacidades perceptuales, lesión cerebral, disfunción cerebral mínima, dislexia, y afasia del desarrollo" (Nasser, 2013).

Con respecto a las dificultades de leer y escribir, en la etapa escolar los estudiantes entre edades de 6 a 8 años pueden presentar problemas en el desarrollo de la escritura y la habilidad psicomotora y problemas de lateralidad (Egido Ramos, 2014).

Por otro lado este tipo de dificultades genera en los padres dificultades, porque generalmente no hay indicios de que sus hijos tengan algún problema psicomotriz y se dan cuenta, es porque algo pasa con su nivel académico (Pallarés, 2012). Cuando se detecta que hay una deficiencia, surge otra dificultad, es que las entidades de salud no cuentan con suficiente personal especializado en el campo, adicional a la falta de cubrimiento de este tipo de problemas en los planes de salud.

Se han realizado diversos estudios acerca de la dyslexia y el impacto que pueden tener en el desarrollo de sistemas computacionales o aplicaciones que permitan mejorar las habilidades psicomotrides de los niños, por ejemplo Vidarte et al. (J.A., M., \& Giráldez, 2009) tuvo como objetivo caracterizar el perfil psicomotor de los niños diagnosticados clínicamente con este déficit, haciendo comparaciones del comportamiento con niños identificados como sanos de su misma edad. El estudio se realizó para un total de 846 niños en el que se obtuvo un porcentaje significativo en el trastorno de atención e hiperactividad. Para ambos casos se hizo el estudio observando habilidades como tonicidad, equilibrio, lateralidad, noción del cuerpo y estructuración del espacio.
Por su parte Sierra (Téllez-de-Reyes, 2014) afirma que la psicomotricidad tiene influencia junto con la educación en el ámbito pedagógico. El propósito del estudio realizado por Sierra consistió en elaborar estrategias educativas útiles para los docentes, en las que se puediera identificar de manera rápida si un estudiante presenta alguna deficiencia. Como resultados se pudo concluir que al presentar el estudiante problemas motrices, el juego libre y espontáneo le permitiría un adecuado tratamiento. De igual forma es de resaltar que la investigación menciona que a través de dinámicas corporales se pueden generar avances cognitivos en los niños. De igual manera, se han realizado trabajos relacionados con el fin de desarrollar e implementar herramientas tecnológicas para estudiantes con dificultades, (Jokisuu, Langdon, \& John, 2011), (LancherosCuesta, Carrillo-Ramos, \& Pavlich-Mariscal, 2014), (Nasser, 2013), (Zhong, Li, Tu, Zhao, \& Shaoqing, 2016).

Teniendo en cuenta lo anterior el presente artículo muestra el desarrollo de Plataforma computacional para la generación de actividades educativas a través de la herramienta Mimio Teach.

En el segundo aparte se describe el diseño de la plataforma, seguido del detalle en la validación. La sección cuarta por su parte muestra una análisis de resultados y por último se mencionan unas conclusiones.

\section{Diseño de la Plataforma}

La plataforma desarrollada incluye un sistema de recomendaciones, que permite la adaptación de las actividades (juegos) teniendo en cuenta el desempeño en pruebas, diagnóstico e interacción de los niños, con un tablero digital (Mimio Teach).

La Figura 1 muestra los principales componentes y etapas del sistema.

El sistema de recomendaciones desarrollado, consiste en entregar una o más actividades didácticas al usuario registrado y que ha realizado la prueba diagnóstica previamente según el resultado de 
acuerdo a los cuatro temas evaluados, el sistema determinará en cuál de estos se requiere mayor atención para así mismo enviarle la actividad que mejor se adapte a sus necesidades. Se tiene en cuenta que el evaluador por su parte, genera una calificación de cada uno de los juegos propuestos, el sistema tendrá en cuenta dicha calificación al recomendar la actividad como prelación, sino entregará una de manera aleatoria.

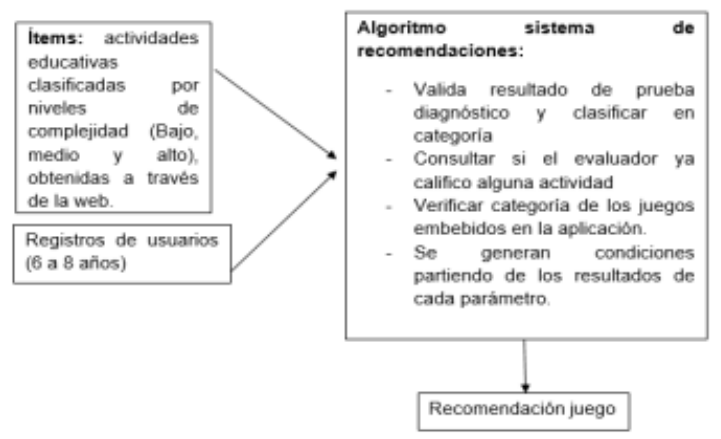

Fig. 1 Componentes del sistema de recomendaciones en la plataforma.

El usuario en su inicio de sesión, debe seleccionar la persona que esté a cargo de su proceso, ya que, si existe más de un evaluador, la aplicación hará el proceso de acuerdo al seleccionado. La plataforma contiene una base de datos con el fin de almacenar la información de forma estructurada, la Figura 2 muestra el diagrama Entidad - Relación.

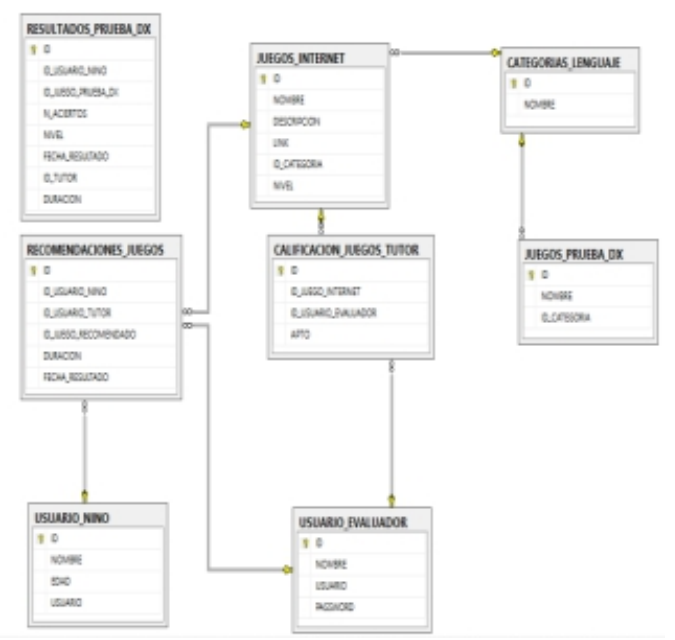

Fig. 2 Diagrama Entidad - Relación.
Con respecto a la interfaz, la Figura 3 muestra el diagrama de navegación y las opciones de la plataforma.

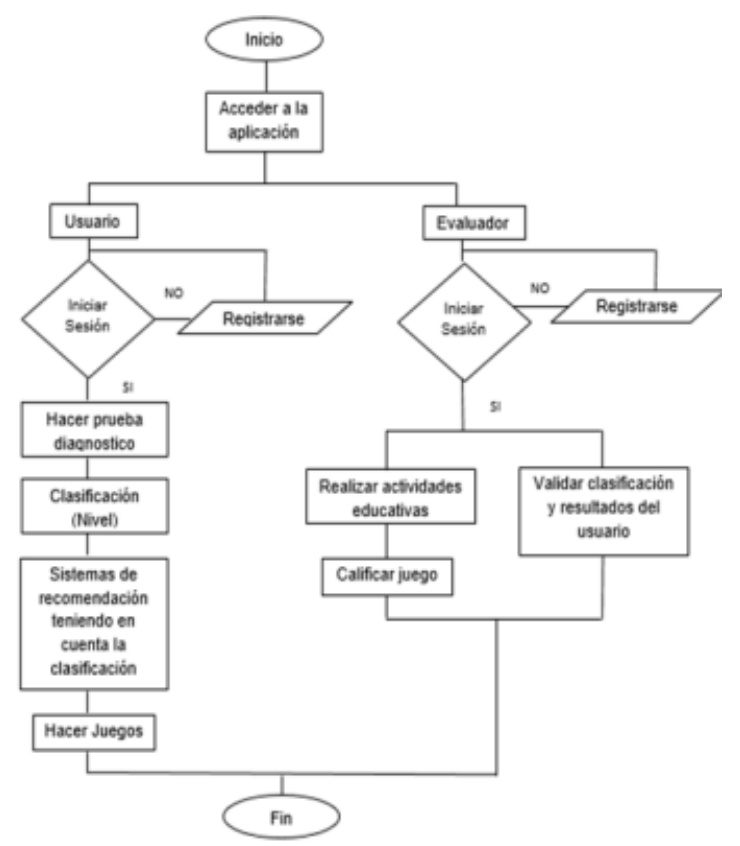

Fig. 3 Diagrama de navegación.

A continuación, se describen las validaciones realizadas.

\section{Validación de la Plataforma}

La implementación de la plataforma desarrollada se realizó a través de una experimentación, en el curso Segundo de Primaria del Colegio La Despensa, sede Marco Fidel Suárez, ubicada en Soacha Cundinamarca, con 32 estudiantes. La actividad se realizó en compañía de un experto en el área de psicología.

La validación tuvo dos momentos, en el primero de ellos se realizaba un diagnostico a través de la plataforma, luego los estudiantes realizaban las actividades con el tablero digital (Mimio Teach), las cuales eran sugeridas por el sistema de recomendaciones. En la Figura 4 se muestra un estudiante participante en las pruebas de diagnóstico. 


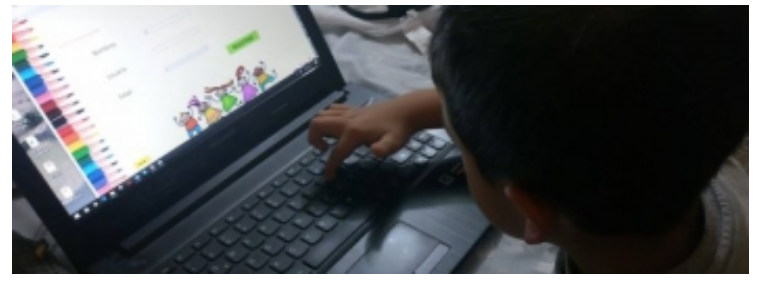

Fig. 4 Estudiantes en la prueba de diagnóstico.

En la Tabla 1, se visualizan los resultados, realizando la respectiva clasificación por niveles, partiendo de los aciertos registrados para cada una de las secciones que integran la prueba y el tiempo total para completarla. Luego de realizar el diagnostico, se realizó una serie de pruebas para observar la funcionalidad existente en el sistema interactivo de Mimio Teach y el sistema de recomendaciones de la plataforma.

La Figura 5 muestra como los niños ingresan en su rol de usuario en la Plataforma. En la Figura 6, se observa como uno de los usuarios interactúa con la aplicación, en este caso se encuentra en la sección de identificación de procesos semánticos.

En la Figura 7, se observa algunos de los juegos realizados por los usuarios según la recomendación brindada por el sistema.

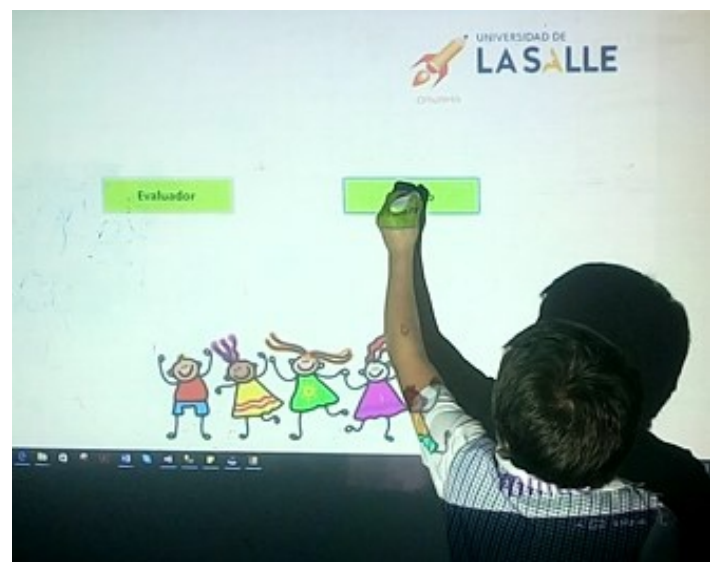

Fig 5. Estudiante accediendo a la plataforma

\section{Análisis de Datos}

Después de realizar el diagnóstico, la docente seleccionó al azar 4 de sus estudiantes para realizar el proceso con la aplicación a través de Mimio Teach. En las Tablas, 1, 2, 3 y 4, se observan los resultados obtenidos, registrados en la misma plataforma, evidenciando su nivel de clasificación en la prueba con el número de aciertos, el tiempo por sección y el tiempo total.

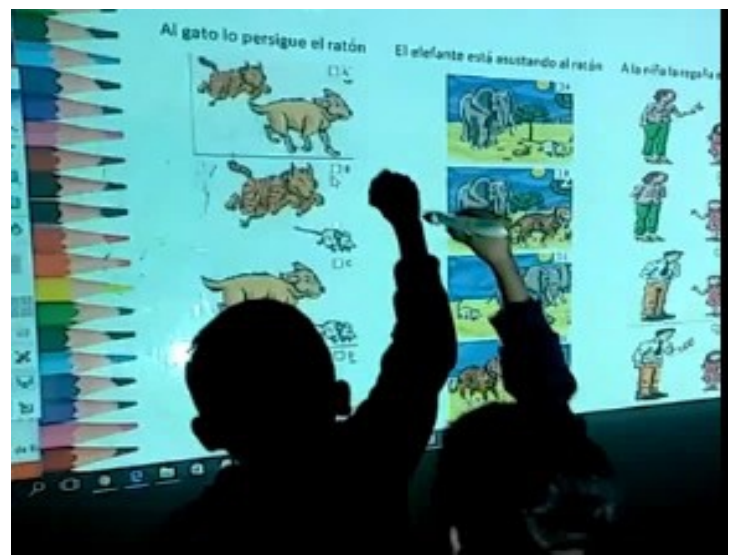

Fig. 6. Estudiantes interactuando con las actividades de lección.
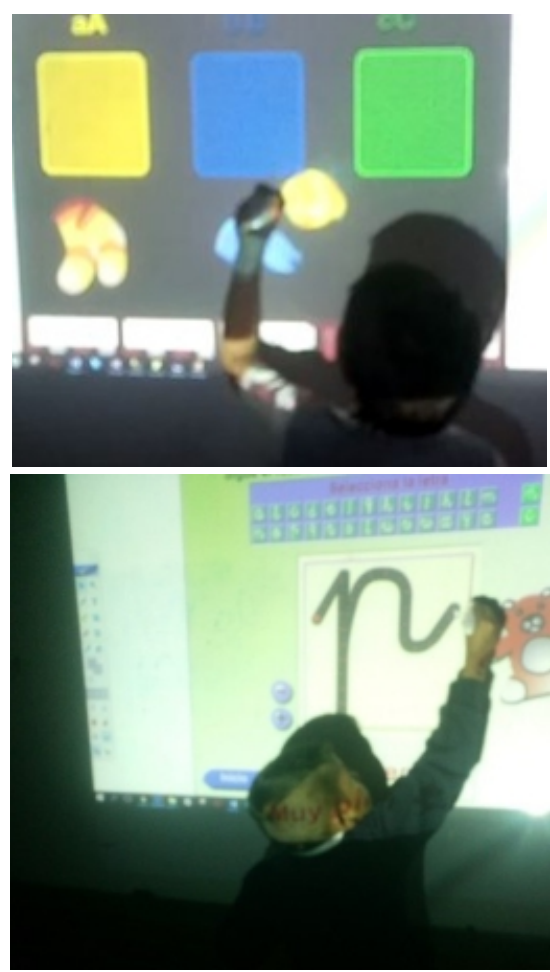

Fig. 7. Estudiantes interactuando con actividades recomendadas. 
Tabla 1. Usuario $N^{\circ} 1$ evaluado ( 6 años)

\begin{tabular}{|l|l|c|l|l|}
\hline Categoría Prueba & Nivel & Aciertos & Duración & Tiempo total prueba \\
\hline Identificación de letras & Medio & 4 & $5 \mathrm{~m} 51 \mathrm{~s}$ & \multirow{2}{*}{$10 \mathrm{~m} 46 \mathrm{~s}$} \\
\hline Procesos Léxicos & Alto & 9 & $1 \mathrm{~m} \mathrm{39} \mathrm{s}$ & \\
\hline Procesos gramaticales & Alto & 3 & $1 \mathrm{~m} 50 \mathrm{~s}$ & \\
\hline Procesos semánticos & Medio & 2 & $1 \mathrm{~m} 26 \mathrm{~s}$ & \\
\hline
\end{tabular}

Tabla 2. Usuario $\mathrm{N}^{\circ} 2$ evaluado (7 años)

\begin{tabular}{|c|c|c|c|c|}
\hline Categoría Prueba & Nivel & Aciertos & Duración & Tiempo total prueba \\
\hline Identificación de letras & Medio & 3 & $6 \mathrm{~m} 34 \mathrm{~s}$ & \multirow{4}{*}{$12 \mathrm{~m} 55 \mathrm{~s}$} \\
\hline Procesos Léxicos & Alto & 10 & $2 \mathrm{~m} \mathrm{20s}$ & \\
\hline Procesos gramaticales & Alto & 3 & $1 \mathrm{~m} 10 \mathrm{~s}$ & \\
\hline Procesos semánticos & Medio & 2 & $2 \mathrm{~m} 51 \mathrm{~s}$ & \\
\hline
\end{tabular}

Tabla 3. Usuario $N^{\circ} 3$ evaluado (8 años)

\begin{tabular}{|c|c|c|c|c|}
\hline Categoría Prueba & Nivel & Aciertos & Duración & Tiempo total prueba \\
\hline Identificación de letras & Bajo & 0 & $0 \mathrm{~m} \mathrm{51s}$ & \multirow{4}{*}{$7 \mathrm{~m} 37 \mathrm{~s}$} \\
\hline Procesos Léxicos & Alto & 9 & $1 \mathrm{~m} \mathrm{43s}$ & \\
\hline Procesos gramaticales & Alto & 3 & $2 \mathrm{~m} 10 \mathrm{~s}$ & \\
\hline Procesos semánticos & Medio & 2 & $2 \mathrm{~m} 53 \mathrm{~s}$ & \\
\hline
\end{tabular}

Tabla 4. Usuario $\mathrm{N}^{\circ} 4$ evaluado (8 años)

\begin{tabular}{|c|c|c|c|c|}
\hline Categoría Prueba & Nivel & Aciertos & Duración & Tiempo total prueba \\
\hline Identificación de letras & Medio & 3 & $3 \mathrm{~m} \mathrm{33s}$ & \multirow{4}{*}{$8 \mathrm{~m} 10 \mathrm{~s}$} \\
\hline Procesos Léxicos & Bajo & 3 & $2 \mathrm{~m} \mathrm{21s}$ & \\
\hline Procesos gramaticales & Medio & 3 & $1 \mathrm{~m} \mathrm{42s}$ & \\
\hline Procesos semánticos & Bajo & 1 & $0 \mathrm{~m} 34 \mathrm{~s}$ & \\
\hline
\end{tabular}

Con los resultados obtenidos y el pilotaje realizado, se pudo evidenciar con ayuda de la docente, que, a través de la herramienta, ella pudo detectar que el tiempo de ejecución en cada sección, puede mostrar efectivamente quienes tienden a confundir las letras (b, d, q) u oraciones.

Por medio de los juegos recomendados, se puede reforzar el tema en el que se detecta mayor dificultad. Adicionalmente, es interesante que este tipo de tecnologías se pudiesen integrar en los colegios oficiales, por ejemplo, en esta institución, no hay el suficiente tiempo para poder adaptar las herramientas a las clases, pero son muy valiosas porque son actividades que llaman la atención de los niños y así demuestran mucho más interés.

Considera que por medio de este sistema se trabaja la motricidad fina y viso-motora, por el hecho de manejar el lápiz; en algunos casos los adultos pueden presentar mayor dificultad para trabajar con este tipo de nuevas tecnologías a diferencia de los niños que se adaptan más rápidamente. Con Mimio Teach sintió que sus estudiantes se sintieron más atraídos porque nunca lo habían utilizado, al hacer este proceso en papel o un computador no lo realizarían con la misma actitud. Los docentes expresaron que sería muy importante tener la oportunidad de capacitarse en el uso de estas herramientas, ya que de esta manera ellos podrían generar estrategias para facilitar la lectura y escritura de sus estudiantes.

Según la psicóloga que realizó acompañamiento en el proceso, consideró que es importante tener en cuenta el contexto ambiental en el cual están sumergidos los sujetos evaluados con la herramienta, debido a que eso en gran medida afecta en el desenvolvimiento y en los resultados de los niños en las pruebas técnicas. Por otra parte, los contextos sociales, económicos, culturales, educativos $\mathrm{y}$ familiares se deben tener en cuenta debido a que, si un niño está realizando la actividad frente a un compañero, con el cual presenta rivalidad, la presión será más fuerte para el, o si el docente que lo acompaña es con el que menos interactúa, el sujeto no tendrá deseos de divertirse como si fuera un juego, que es el objetivo de la actividad.

\section{Conclusiones}

Mimio Teach (Tablero Digital) es un sistema interactivo enfocado al ámbito educativo, que permitió la adaptabilidad con la aplicación diseñada, donde los usuarios muestran interés, al ser una herramienta tecnológica e innovadora, que permite medir y trabajar en la mejora de las dificultades detectadas, aprendiendo mientras se divierte y enfocados en la parte psicomotriz fina. Es importante reconocer la edad seleccionada que es de 6 a 8 años ya que en ella pueden hacer manipulación de la herramienta de manera acorde con su repertorio conductual, es decir que su forma de ser ya está definida. Los sistemas de recomendaciones hoy día se pueden aplicar en diferentes áreas, en este proyecto se puede observar como contribuyen el desarrollo de algoritmos en la educación, ya que, partiendo de las habilidades y capacidades del usuario, se brinda una recomendación para que comience un proceso correctivo. 
Lancheros Cuesta, D., \& Vargas, M. D. (2019). Plataforma computacional para la generación de actividades educativas a través de la herramienta Mimio Teach. Teknos Revista Científica, 19(2), 35-40.

\section{Referencias}

Nasser, K. (2013). Diagnosing Learning Disabilities in a Special Education By an Intelligent Agent Based System. International Journal of Advanced Computer Science and Applications.

Egido Ramos, B. (2014). Las dificultades de la lectoescritura: dislexia y disgrafía. pautas de intervención y estudio de un caso en educación primaria. Universidad de Valladolid.

Pallarés, A. (2012). Problemas asociados a la dislexia. Revista Neurológica.

J.A., V., M., E., \& Giráldez, M. (2009). Perfil psicomotor de niños de 5 a 12 años diagnosticados clínicamente de trastorno por déficit de atención/hiperactividad en Colombia. Obtenido de https://www.neurologia.com/articulo/2008619: https://www.neurologia.com/articulo/2008619

Téllez-de-Reyes, M. C. (2014). Estudio de la psicomotricidad de niños y niñas en edad preescolar. Obtenido de UNIR: https://reunir.unir.net/handle/123456789/2401

Jokisuu, E., Langdon, P., \& John, C. (2011). Modelling Cognitive Impairment to Improve Universal Access. Lecture Notes in Computer Science, 6766.

Lancheros-Cuesta, D., Carrillo-Ramos, A., \& PavlichMariscal, J. (2014). Content adaptation for students with learning difficulties: Design and case study. International Journal of Web Information Systems, 10 (2), 106-130.

Zhong, T., Li, X., Tu, X., Zhao, S., \& Shaoqing, Z. (2016). The prediction of attentional status with task difficulty based on EEG signals. Computer and Communications (ICCC), 2016 2nd IEEE International Conference on.

Al-barrak, L., Kanjo, E., \& Younis, E. M. (2017). NeuroPlace: Categorizing urban places according to mental states. PLOS ONE. 